\title{
Combination of $A \beta$ Suppression and Innate Immune Activation in the Brain Significantly Attenuates Amyloid Plaque Deposition
}

\author{
Christophe Verbeeck, ${ }^{*}$ Anna Carrano, ${ }^{\star}$ Paramita Chakrabarty, ${ }^{\dagger}$ Joanna L. Jankowsky, ${ }^{\ddagger}$ and Pritam Das*
}

\begin{abstract}
From the Department of Neuroscience, ${ }^{*}$ Mayo Clinic College of Medicine, Jacksonville, Florida; the Department of Neuroscience, ${ }^{\dagger}$ Center for Translational Research in Neurodegenerative Disease, McKnight Brain Institute, University of Florida, Gainesville, Florida; and the Department of Neuroscience, ${ }^{\ddagger}$ Baylor College of Medicine, Houston, Texas
\end{abstract}

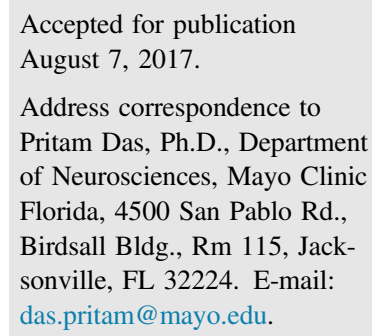

\begin{abstract}
Anti-A $\beta$ clinical trials are currently under way to determine whether preventing amyloid deposition will be beneficial in arresting progression of Alzheimer disease. Both clinical and preclinical studies suggest that antiamyloid strategies are only effective if started at early stages of the disease process in a primary prevention strategy. Because this approach will be difficult to deploy, strategies for secondary prevention aimed at later stages of disease are also needed. In this study, we asked whether combining innate immune activation in the brain with concurrent $A \beta$ suppression could enhance plaque clearance and could improve pathologic outcomes in mice with moderate amyloid pathologic disorder. Starting at 5 months of age, tet-off amyloid precursor protein transgenic mice were treated with doxycycline (dox) to suppress further amyloid precursor protein/A $\beta$ production, and at the same time mice were intracranially injected with adeno-associated virus 1 expressing murine IL-6 (AAV1-mIL-6). Three months later, mice treated with the combination of $A \beta$ suppression and AAV1-mIL- 6 showed significantly less plaque pathologic disorder than dox or AAV1-mIL- 6 only groups. The combination of AAV1-mIL- $6+$ dox treatment lowered total plaque burden by $>60 \%$ versus untreated controls. Treatment with either dox or AAV1-mIL-6 alone was less effective than the combination. Our results suggest a synergistic mechanism by which the up-regulation of mIL- 6 was able to improve plaque clearance in the setting of A $\beta$ suppression. (Am J Pathol 2017, 187: 2886-2894; https://doi.org/10.1016/j.ajpath.2017.08.010)
\end{abstract}

The amyloid hypothesis of Alzheimer disease (AD) postulates that $A \beta$ accumulation in the brain triggers a pathologic cascade that leads to neurodegeneration and progressive cognitive impairment. ${ }^{1-3}$ Recent biomarker and amyloid positron emission tomography imaging studies suggest that brain $A \beta$ amyloidosis may begin several decades before overt neurodegeneration and cognitive deficits. ${ }^{4,5}$ From these observations, significant time and effort has been devoted in the development of anti-A $\beta$ therapies. Numerous studies of anti-A $\beta$ immunotherapy have shown efficacy in preventing amyloid deposition in AD mouse models (reviewed in Golde et $\mathrm{al}^{6,7}$ ); however, when advanced to human clinical trials, these approaches have largely failed to stabilize or improve cognitive function. ${ }^{7-11}$ These failures have led to the assumption that by the time patients are clinically diagnosed with $\mathrm{AD}$, it may be too late for anti-A $\beta$ therapies to be effective. ${ }^{3,7}$ Given that timing is critical, prevention trials are now under way in genetically identified at-risk patients (APOE4 and PSEN1 E280A carriers) and in cognitive normal subjects with preexisting amyloid pathologic disorder (A4 study ${ }^{12}$ ). Promising results were recently reported in a phase $\mathrm{Ib}$ trial of anti-A $\beta$ antibody aducanumab in patients with mild disease, showing reductions of amyloid signal in the brain and some cognitive benefit. ${ }^{13}$ Despite this success, clinical failures continue to beset the AD field, because Merck \& Co (Kenilworth, NJ) recently halted their trial of $\beta$-site amyloid precursor protein enzyme (BACE) 1 inhibitor verubecestat in mild-to-moderate $\mathrm{AD}$ for lack of

Supported by NIH grant RO1AG32991 (to P.D.).

C.V. and A.C. contributed equally to this work.

Disclosures: None declared. 
efficacy. ${ }^{14}$ Clearly, more aggressive or novel treatment strategies are needed for patients with moderate or advanced disease. $^{15}$

We had previously demonstrated that innate immune activation in the brain by overexpression of mIL- 6 and other cytokines can significantly attenuate amyloid deposition in amyloid precursor protein (APP) mouse models. ${ }^{16-18}$ These studies suggested that cytokine up-regulation enhanced microglial-mediated $A \beta$ clearance to attenuate amyloid deposition only when initiated in young APP mice before amyloid deposition. Immune activation was far less effective in reducing pathologic disorder when tested after plaque onset. ${ }^{16}$ We hypothesized that a combination approach may offer improved efficacy at these stages when considerable pathologic disorder already exists. Here, we tested whether combining immune activation with $\mathrm{A} \beta$ reduction would be more effective than either treatment alone in mice harboring moderate preexisting plaque load. We took advantage of the tet-off APP transgenic line to genetically suppress APP/A $\beta$ production mid-life and simultaneously induced microglial activation with the use of adeno-associated virus (AAV)1mediated murine (m)IL- 6 overexpression. After 3 months of treatment, mice were harvested for histopathologic analysis to determine how the combination approach compared with $\mathrm{A} \beta$ suppression or AAV-mIL-6 treatment alone.

\section{Materials and Methods}

Mice

APP transgenic mice, under the control of the tet-off promoter (tet off-APPswe/ind, line 102) were used for these studies. ${ }^{19}$ The tet off-APPswe/ind mice were mated to mice expressing tetracycline transactivator (tTA) under the control of the $\mathrm{Ca}^{2+} /$ calmodulin-dependent kinase II $\alpha$ promoter-tTA line B. ${ }^{20}$ Male APP/tTA double-transgenic mice were then mated with wild-type Friend leukemia virus B female mice to generate experimental mice for these studies ( $n=5$ to 8 females per group). All animal procedures were approved by the Mayo Clinic Institutional Animal Care and Use Committee.

\section{Doxycycline Administration}

Doxycycline (dox) treatment was started at 5 months of age and continued for 3 months until harvest at 8 months of age. Dox was formulated in mouse chow (Purina 5001 chow; Bio-Serv, Flemington, NJ) at a concentration of $50 \mathrm{mg} / \mathrm{kg}$.

\section{AAV1 Preparation and Hippocampal Injections}

AAV1 expressing mIL-6 or enhanced green fluorescent protein (EGFP) under the control of the cytomegalovirus enhancer/ chicken $\beta$-actin promoter was generated and injected in both sides of the hippocampus as described previously. ${ }^{16}$ For stereotactic injections, mice ( $n=5$ to 8 females per group) were anesthetized with $1.5 \%$ isoflurane in $1 \%$ oxygen and secured into a Kopf apparatus (Model 900 Small Animal Stereotactic Instrument; David Kopf Instruments, Tujunga, CA). The coordinates for hippocampal injection were -1.7 caudal, -1.6 lateral, and -1.2 ventral from the bregma. A UMP2 Microsyringe Injector and Micro4 Controller were used to inject $2 \mu \mathrm{L}$ of AAV (AAV1-mIL6 at 7.10E+12 viral particles/hippocampus and AAV1-EGFP at 6.3E+12 viral particles/hippocampus) at a constant rate over a 10-minute period. After an additional 10 minutes were allowed, the needle was raised slowly, the scalp incision was closed aseptically, and mice were revived under a heating lamp.

\section{Immunoblotting, Cytokine ELISA, and A $\beta$ ELISA}

For measurements of $A \beta$ levels in mice, frozen left hemi brains were sequentially extracted in Tris-buffered saline (TBS), TBS buffer containing $1 \%$ Triton X-100 (TBSx), and $5 \mathrm{~mol} / \mathrm{L}$ guanidine in $50 \mathrm{~mm}$ Tris- $\mathrm{HCl}, \mathrm{pH} 8.0$, as described previously. ${ }^{21} \mathrm{~A} \beta$ levels from forebrain lysates (cortex + hippocampus combined) of APP/tTA mice were measured biochemically using human $\mathrm{A} \beta$ end-specific sandwich enzyme-linked immunosorbent assay (ELISA) as described previously. ${ }^{21}$ IL- 6 cytokine levels were analyzed from TBSsolubilized forebrain lysates (cortex + hippocampus combined) using BD OptiEIA ELISA kits (BD Biosciences Pharmingen, San Diego, CA). To detect APP levels, TBSxextracted protein samples were separated on Bis-Tris $12 \%$ XT gels (Bio-Rad, Hercules, CA), then probed with CT20 antibody (dilution 1:1000; Mayo Clinic, Jacksonville, FL) as previously described. ${ }^{21}$ Relative band intensity was quantified using ImageJ software version 1.43 (NIH, Bethesda, MD).

\section{Immunohistochemical Staining and Image Processing}

After tissue harvesting, the right hemi brain was fixed in $4 \%$ paraformaldehyde for 24 hours, paraffin embedded, and $5-\mu \mathrm{m}$ sections were used for immunostaining using the following antibodies as described before ${ }^{21}$ : pan-A $\beta$ antibody 33.1.1 (dilution 1:1500; Mayo Clinic), A $\beta$ 1-40 specific monoclonal antibody (mab) 13.1.1 (dilution 1:500, Mayo Clinic), glial fibrillary acidic protein (GFAP; dilution 1:1000; Sigma-Aldrich, St. Louis, MO), Iba-1 (dilution 1:1000; Wako, Richmond, VA), and EGFP (dilution 1:1000; Invitrogen, Carlsbad, CA). Target antigen retrieval was performed by steaming the sections for 30 minutes in deionized water. Endogenous peroxidase was blocked for 5 minutes with $0.03 \%$ hydrogen peroxide. Sections were then blocked with 5\% normal goat serum for 20 minutes. Subsequently, sections were incubated 1 hour at room temperature in primary antibodies, then incubated in secondary antibodies (Envision-Plus-labeled polymer-horseradish peroxidase; Dako, Carpentaria, CA), for 30 minutes at room temperature. Peroxidase labeling was visualized with the chromogen solution 3, 3'-diaminobenzidine. Sections immunostained for microglia (Iba1) and astrocytes (GFAP) 
A

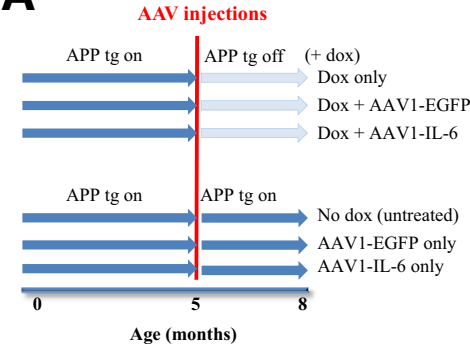

B

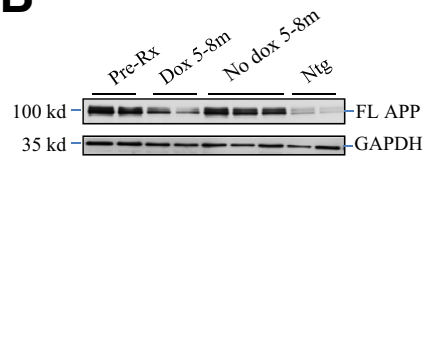

C

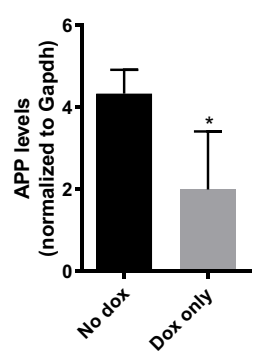

D $\quad$ EGFP

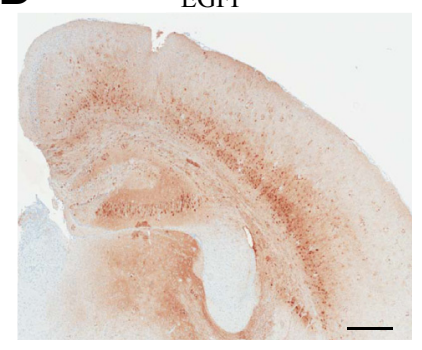

Figure 1 Experimental design for combination treatment using amyloid precursor protein (APP)/Aß suppression and innate immune activation. A: Experimental design. 5-month-old APP/tetracycline transactivator (tTA) transgenic mice with moderate amyloid deposition throughout the forebrain were treated with doxycycline (dox; $50 \mathrm{mg} / \mathrm{kg}$ chow concentration) to halt further expression of transgenic APP/A $\beta$ for 3 months. At the same time, mice were intracranially injected with adeno-associated virus (AAV)1 expressing murineIL-6 (mIL-6; experimental) or expressing enhanced green fluorescent protein (EGFP; control) to target the hippocampus. These combination treatment groups were sacrificed at 8 months of age to determine effect on amyloid deposition. Additional experimental groups include i) untreated mice (no dox, no AAV, harvested at 8 months of age), ii) dox only, and iii) AAV1-mIL-6 only. B: Top panel: Representative Western blot analysis probed with anti-CT20 antibody showing full-length APP in untreated controls or after 3 months of dox treatment. Bottom panel: Glyceraldehyde-3-phosphate dehydrogenase (GAPDH) levels from the same blot. C: Quantitative densitometric analysis of APP expression (normalized to GAPDH) shows an approximately 50\% reduction in dox-treated mice. D: Representative brain section immunostained with anti-EGFP antibody shows widespread EGFP expression in the forebrain of AAV1-EGFP-injected mice. $n=5$ to 8 female mice per group (A). ${ }^{*} P<0.05$ versus no dox. Scale bar $=1 \mathrm{~mm}$. FL, full-length; $\mathrm{tg}$, transgenic; Rx, therapy.

were further stained with $1 \%$ Congo red (Sigma-Aldrich) to visualize amyloid plaques. Additional sections were also stained with $1 \%$ Thioflavin S (Thio-S, practical grade; Sigma-Aldrich) to visualize amyloid plaques. Immunostained sections were captured using the Scanscope XT image scanner (Aperio Technologies, Vista, CA). A $\beta$ plaque burden in the cortex and hippocampus was calculated using the Positive Pixel Count Program (Imagescope software, version 12.3; Aperio Technologies) in sections immunostained with pan-A $\beta 33.1 .1$ or $\mathrm{A} \beta$ 1-40-specific mab 13.1.1 antibody. At least three sections per mouse brain $(n=5$ to 8 mice per group) were used to calculate the average plaque burden in the hippocampus and cortex for each sample. All of the above analyses were performed in a blinded manner (C.V., and A.C.). Thio-S quantitation (mean area and integrated density in the cortex and hippocampus) was performed using ImageJ.

\section{Quantitative RT-PCR}

Total RNA from mice hippocampus was extracted using the RNeasy mini kit (Qiagen, Valencia, CA) according to the manufacturer's protocol. Total RNA was dissolved in nuclease-free water and stored at $-80^{\circ} \mathrm{C}$. Reverse transcription was performed using Superscript III (Invitrogen) with the use of Mastercycler pro, and the reaction mix was subjected to quantitative RT-PCR with the use of iQ SYBR Green Supermix (Bio-Rad) to detect the amplification products. Relative quantification of mRNA expression was calculated by the $\Delta \mathrm{C}_{\mathrm{T}}$ method after adjusting the levels to the corresponding internal glyceraldehyde-3-phosphate dehydrogenase control for each sample.

The sequences of primers used to amplify target genes by quantitative RT-PCR were as follows: Gapdh [5'-AGGTCGGTGTGAACGGATTTG-3' (forward) and 5'-TGTAGACCATGTA-
GTTGAGGTCA-3' (reverse)], Aifl [5'-CTTGAAGCGAATGCTGGAGAA- $3^{\prime}$ (forward) and 5'-GGCAGCTCGGAGATAGCTTT-3' (reverse)], Itgam [5'-GTGTGACTACAGCACAAGCCG-3' (forward) and 5'-CCCAAGGACATATTCACAGCCT-3' (reverse)], C $x 3 \operatorname{crl} 1$ [5'-ACCGGTACCTTGCCATCGT-3' (forward) and 5'-ACACCGTGCTGCACTGTCC-3' (reverse)], Trem2 [5'-GCCTTCCTGAAGAAGCGGAA- $3^{\prime}$ (forward) and $5^{\prime}$ GAGTGATGGTGACGGTTCCA-3' (reverse)], Hexb [5'ACTCCAAGATTATGGCCTCGAGCA- $3^{\prime}$ (forward) and $5^{\prime}$-GCTATTCCACGGCTGACCATTCT-3' (reverse)], and Gfap [5'-ACCAGCTTACGGCCAACAG- $3^{\prime}$ (forward) and $5^{\prime}$-CCAGCGATTCAACCTTTCTCT- $3^{\prime}$ (reverse)].

\section{Statistical Analysis}

Two-way analysis of variance with Tukey's multiplecomparison post hoc test or 2-tailed t-test was used for statistical comparison (GraphPad Prism 6 Software; GraphPad, San Diego, CA).

\section{Results}

Combining $A \beta$ Suppression with Innate Immune Activation Significantly Attenuates Amyloid Deposition in Aged APP/tTA Mice

All treatments were started at 5 months of age to ensure that APP/tTA mice would already have developed a moderate amyloid load throughout the cortex before any intervention. A schematic for the experimental design, including all treatment groups, is provided in Figure 1A. Mice used for combination treatment were fed with dox $(50 \mathrm{mg} / \mathrm{kg}$ in chow) to suppress further expression of transgenic APP/A $\beta$ by approximately $50 \%$ versus untreated (no dox) mice (Figure 1, B and C). At the same age, combination treatment 

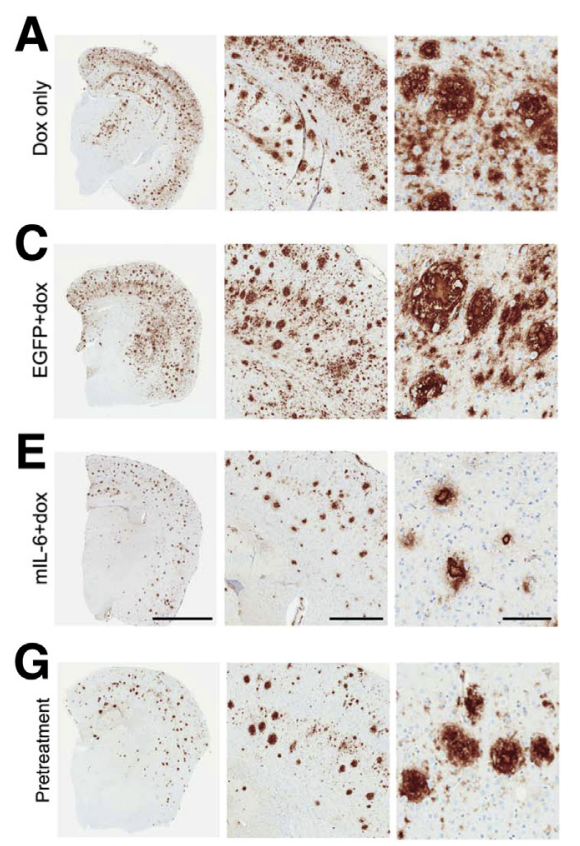

I

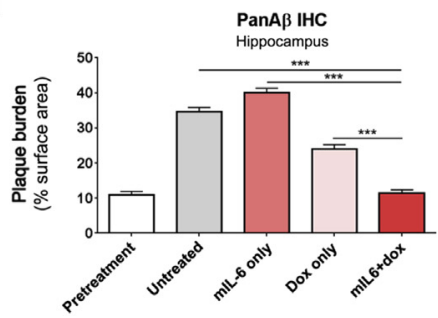

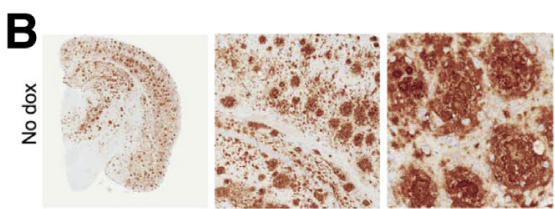
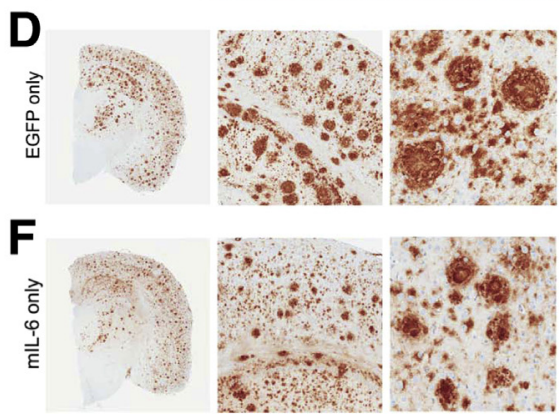

H
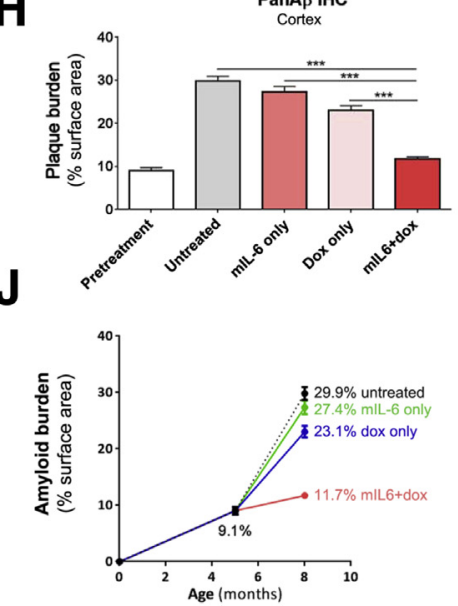

Figure 2 Combination treatment significantly attenuates amyloid deposition in amyloid precursor protein (APP)/tretracycline transactivator (tTA) mice. A-G: Representative brain sections immunostained with pan-A $\beta$ antibody (33.1.1) show amyloid plaque staining throughout the forebrain of APP/tTA mice treated with doxycycline (dox) only (A), untreated (B), adeno-associated virus 1 (AAV1)-enhanced green fluorescent protein (EGFP) + dox (C), AAV1-EGFP only (D), AAV1 expressing murine IL-6 (AAV1-mIL-6) + dox (E), AAV1-mIL-6 only (F), and pretreatment (at 5 months of age) (G). Combination treatment with AAV1-mIL-6 + dox (C) shows less amyloid staining, particularly for diffuse $A \beta$ plaques both surrounding cored plaques and in the remaining brain parenchyma. $\mathbf{H}-\mathbf{I}$ : Plaque quantification from $A \beta$ immunostained sections (cortex; H) and (hippocampus; I) confirmed a significant decrease in amyloid deposition in the combination AAV1-mIL$6+$ dox group versus control groups. J: Combination treatment approach is more effective than either approach alone. The combination treatment group (mIL-6 + dox), showed a robust and significant attenuation of plaque deposition versus $A \beta$ suppression alone (dox only) or AAV-mIL-6 alone. ${ }^{* *} P<0.001$. Scale bars: $2 \mathrm{~mm}(\mathbf{A}-\mathbf{G}$, left column); $500 \mu \mathrm{m}(\mathbf{A}-\mathbf{G}$, middle column); 100 $\mu \mathrm{m}(\mathbf{A}-\mathbf{G}$, right column). IHC, immunohistochemistry. mice were administered recombinant AAV1-mIL-6 by stereotaxic intracranial injection to target the hippocampus to promote innate immune activation. When AAV1-IL-6 vector is injected into the mouse brain at postnatal day 0 , this approach produces widespread expression of mIL-6 in the mouse brain. ${ }^{16}$ Here, a control AAV1 was used to deliver EGFP by stereotaxic injection into the adult hippocampus to demonstrate that this approach expresses the gene of interest in the hippocampus and overlying cortical layers (Figure 1D). that the stereotaxic hippocampal injection of AAV1-mIL-6 results were confirmed in more than threefold elevation of mIL-6 protein in the brain as measured by ELISA from TBS-solubilized forebrain lysates (cortex + hippocampus combined): $3435.8 \pm 812 \mathrm{mg} / \mathrm{mL}$ in the AAV-IL-6-injected mice versus $1105.4 \pm 213 \mathrm{mg} / \mathrm{mL}$ in untreated controls.

Animals were harvested at 8 months of age after 3 months of differential treatment. We first evaluated the impact of combining A $\beta$ suppression (dox treatment) with AAV1-mIL6 on amyloid deposition in the brains of APP/tTA mice. Mice treated with AAV1-mIL-6 + dox showed significant attenuation of amyloid deposition in the cortex (11.7\% surface area) (Figure 2, E and $\mathrm{H}$ ) versus untreated controls $(29.9 \%$ surface area) (Figure 2, B and H). APP/tTA mice treated only with dox for the same length of time had a smaller reduction in amyloid deposition (23.1\% surface area) (Figure 2, A and $\mathrm{H}$ ), whereas treatment with AAV1-mIL-6 alone (mIL6only group) had minimal effect on amyloid deposition (27.4\% surface area) (Figure 2, F and H). For comparison, we included two additional control groups: AAV1-EGFP + dox (18.5\% surface area) (Figure 2C) and AAV1-EGFP only (28.3\% surface area) (Figure 2D). Because AAV1-mIL-6 was targeted to the hippocampus, we also assessed $A \beta$ plaque burden in the hippocampus. Once again, the combination of AAV1-mIL-6 significantly reduced amyloid deposition (11.5\% surface area) (Figure 2, E and I) versus untreated controls (34.7\% surface area) (Figure 2, B and I). Hippocampal amyloid burden in the other experimental groups were as follows (Figure 2, A-G and I): dox only $(24.0 \%$ surface area), AAV1-mIL6 only (40.0\% surface area), AAV1-EGFP + dox (18.4\% surface area), and AAV1-EGFP only (37.1\% surface area). ELISA A $\beta$ measurement from guanidine-solubilized forebrain lysates (cortex + hippocampus combined) showed similar results: the concentration of A $\beta 42$ was significantly reduced relative to untreated controls only in mice receiving AAV1-mIL-6 + dox (Table 1). The 
Table 1 A $\beta$ ELISA Showed a Significant Decrease of Guanidine-Extracted A $\beta 42$ in the Combination AAV1-mIL- $6+$ Dox Group versus Control Groups

\begin{tabular}{llllll}
\hline A $\beta$ species & Pretreated & Untreated & mIL-6 only & Dox only & mIL-6 + dox \\
\hline$A \beta 42, \mathrm{pmol} / \mathrm{g}$ & $2301 \pm 302$ & $4973 \pm 402$ & $4327 \pm 394$ & $3947 \pm 280$ & $2764 \pm 377^{*}$ \\
$\mathrm{~A} \beta 40, \mathrm{pmol} / \mathrm{g}$ & $2094 \pm 285$ & $3773 \pm 362$ & $3628 \pm 275$ & $2668 \pm 257$ & $2325 \pm 299$ \\
\hline
\end{tabular}

Data are expressed as means \pm SEM.

${ }^{*} P<0.05$.

AAV1-mIL-6, adeno-associated virus 1 expressing murine IL-6; Dox, doxycycline; ELISA, enzyme-linked immunosorbent assay.

concentration of $\mathrm{A} \beta 40$ also decreased in the AAV1-mIL$6+$ dox group but did not reach statistical significance (Table 1).

\section{Combination Treatment Is More Effective Than A $\beta$ Suppression or AAV1-mIL-6 Treatment Alone}

To further assess the efficacy of combination treatment, we compared amyloid plaque burden in the brains of untreated controls with either single modality (dox only and AAV1mIL-6 only) versus combination treatment (AAV1-mIL$6+$ dox) (Figure $2 J$ ). Immunohistologic analysis of pan-A $\beta$ staining showed that at the start of the experiment, 5-monthold APP/tTA mice harbored a moderate degree of preexisting amyloid in the cortex $(9.1 \%$ surface area). Left untreated, the area of cortical A $\beta$ staining in APP/tTA mice harvested at 8 months of age increased by approximately threefold (29.9\% surface area). Relative to the approximately $30 \%$ burden in untreated mice, administration of AAV1-mIL-6 produced only a small approximately $8 \%$ decrease in amyloid plaque area. Similarly, in the dox-only group, amyloid plaque burden decreased by just approximately $21 \%$. In contrast, treatment with AAV1-mIL$6+$ dox decreased plaque area by approximately $60 \%$ versus untreated controls, showing that combination treatment was significantly more effective in attenuating amyloid plaque deposition than either $A \beta$ suppression or AAV1mIL-6 treatment alone.

We then evaluated whether combination treatment affected dense-core amyloid plaques in the APP/tTA mice with the use of Thio-S histological examination and A $\beta 40$ immunostaining to detect this subset of $A \beta$ deposits. ${ }^{22} \mathrm{APP} /$ tTA mice treated with AAV1-mIL- $6+$ dox showed significant reductions in both measures of dense-core plaque burden (Figure 3) versus untreated controls. Comparable reductions in dense-core plaque load were also seen in the dox-only group and, to a lesser extent, in the mIL-6-only group (Figure 3), suggesting that combination therapy did not promote the clearance of fibrillar plaques beyond the effect attained by $A \beta$ suppression alone.

\section{Microglial Activation by mIL- 6 as a Mediator of $A \beta$ Clearance}

We previously demonstrated that mIL-6 activates both astrocytes and microglia in the cortex and hippocampus of
AAV1-mIL-6-injected APP mice. ${ }^{16}$ mIL-6 overexpression promotes microglial up-regulation of phagocytic markers such as CD11b, suggesting a role for activated microglia in $\mathrm{A} \beta$ clearance. ${ }^{16}$ Consistent with this earlier finding, treatment with AAV1-mIL-6 in the combination group significantly increased the density of Iba-1-positive microglia and GFAP-positive astrocytes. Throughout the hippocampus and cortex, both cell types displayed morphologic changes such as hypertrophy consistent with immune activation in mice given AAV1-mIL6 + dox (Figure 4, A and B). Whereas in dox only and AAV1-EGFP + dox control groups, Iba-1-positive microglia and GFAP-positive astrocytes were predominantly clustered around plaques (Figure 4, A and B). Quantitative real-time PCR confirmed that Aifl mRNA encoding Ibal was increased approximately threefold in the mIL- $6+$ dox group versus dox only and AAV1-EGFP + dox control mice (Figure 4C). Moreover, Itgam mRNA encoding the microglial pattern recognition and professional phagocytic receptor $\mathrm{CD} 11 \mathrm{~b}^{23}$ was increased $>20$-fold, whereas the fractalkine receptor $\mathrm{Cx} 3 \mathrm{crl}$ mRNA was increased $>10$-fold in the mIL- 6 mice + dox group versus the dox-only and AAV1-EGFP + dox control mice (Figure 4C). mRNA for the lysosomal $\beta$-hexosaminidase Hexb and the triggering receptor expressed on myeloid cells 2 Trem $2^{24,25}$ were not significantly altered (Figure 4C).

\section{Discussion}

The failure of multiple recent anti-A $\beta$ clinical trials in patients with mild-to-moderate $\mathrm{AD}$ has prompted discussion of future trials to test preventative treatments. Despite the disappointing results for $A \beta$-lowering therapies when started after the onset of cognitive symptoms, the same approaches remain viable options for $\mathrm{AD}$ prevention, particularly in at-risk patients who harbor amyloid pathologic disorder but are still cognitively normal. This unmet need led us to investigate whether combining two complementary approaches for $A \beta$ reduction might improve efficacy at this stage of preclinical disease. We used a mouse model to test whether lowering $A \beta$ production by suppressing expression of transgenic APP and concurrently enhancing $A \beta$ clearance by activation of the innate immune system in the brain would attain better plaque control than either approach alone. We show that in mice that harbor a moderate plaque burden at the outset of treatment, the 
A
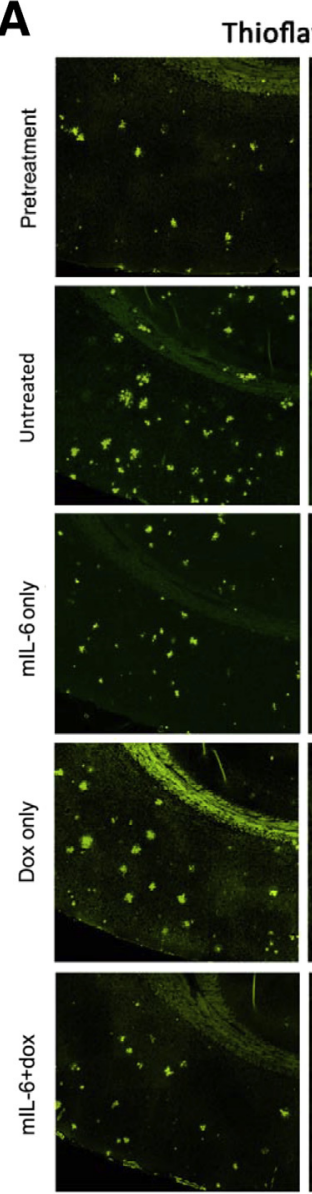

C

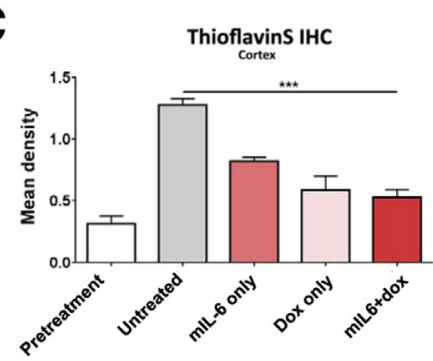

B
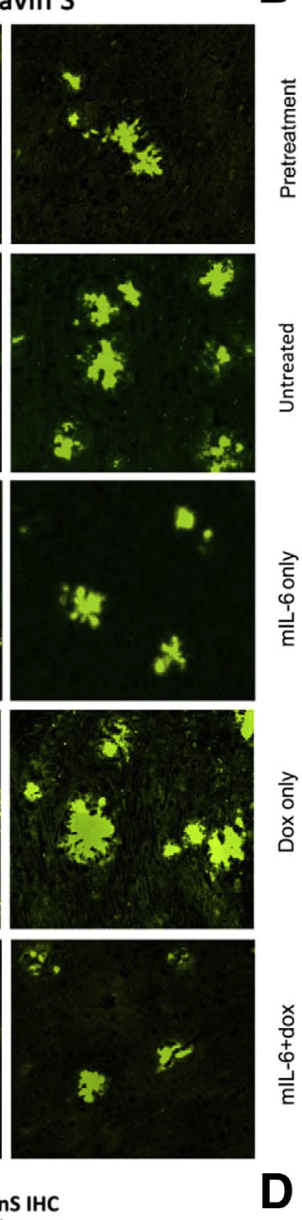

D

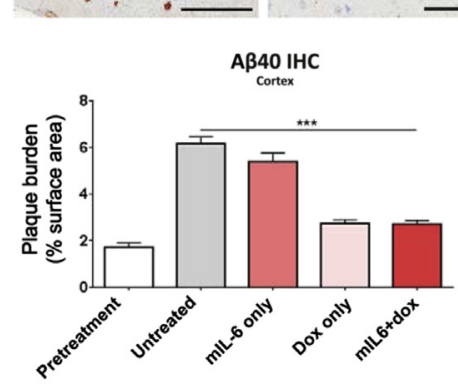

$A \beta 40$

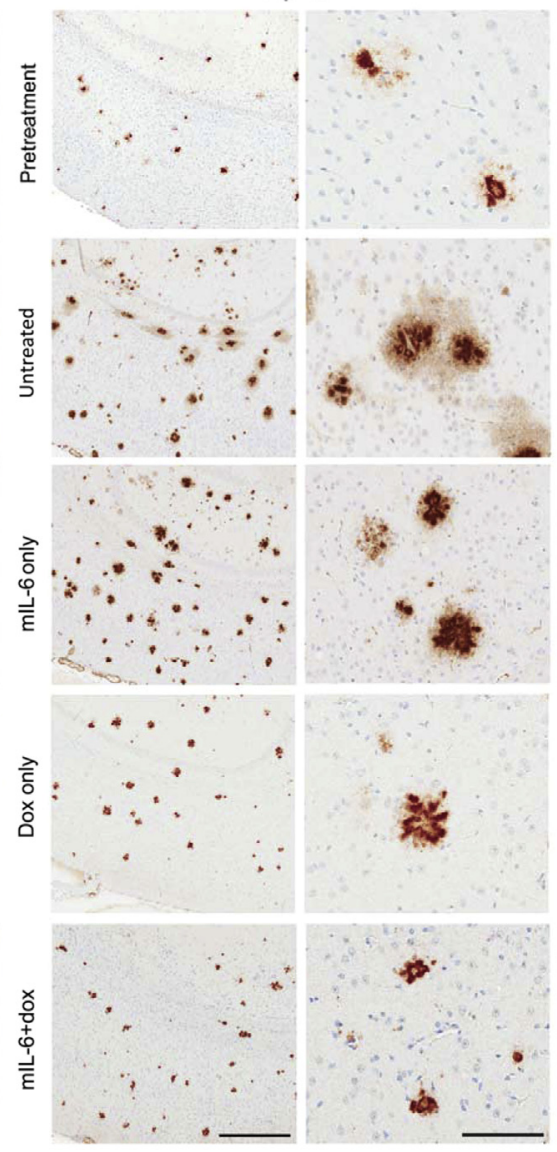

Figure 3 Thioflavine-S-positive amyloid plaques were less responsive to combination therapy than diffuse $A \beta$ deposits. $\mathbf{A}$ and $\mathbf{B}$ : Representative brain sections stained with (A) Thioflavine-S or (B) anti-Aß40 13.1.1 antibody. C and D: Quantification of Thioflavine-S (C) or anti-A $\beta 40$ 13.1.1 -stained plaque load (D) shows a significant decrease in dense-cored plaque load in murine $(\mathrm{m})$ IL-6 + doxycycline (dox) and dox-only groups versus untreated mice. ${ }^{* * *} P<0.001$. Scale bars: $500 \mu \mathrm{m}$ (A and B, left column); $100 \mu \mathrm{m}$ (A and $B$, right column). combination strategy was significantly more effective at preventing further plaque deposition than either treatment alone.

Our present findings support previous work showing that substantial plaque clearance could be attained by combining APP/A $\beta$ suppression with anti-A $\beta$ immunotherapy. ${ }^{26}$ One important difference between the present and previous experiments is the extent to which transgenic APP was suppressed. In our earlier study, we maximally suppressed transgenic APP by $>95 \%$ before adding passive immunotherapy as an adjuvant treatment. ${ }^{26}$ Here, we opted for partial APP reduction by using a lower dosage of doxycycline to attain approximately $50 \%$ transgene suppression. We think that partial transgene reduction more accurately mimicked the effect of A $\beta$-lowering drugs such as BACE1 inhibitors that may someday be used in the clinic. Lowering the amount of dox used for transgene suppression had the added benefit of avoiding any potential interference between the antibiotic and mIL-6-mediated immune activation in the brain.

$\mathrm{We}^{16-18}$ and others ${ }^{27}$ have previously reported that viral-mediated cytokine expression in the brain (eg, IL-6, IL-1 $\beta$, tumor necrosis factor $\alpha$, interfon- $\gamma$ ) from birth significantly attenuates amyloid formation in adult APP transgenic mice. Expression of innate immune cytokines provoked microglial activation to enhance microglial uptake and clearance of $A \beta$. In the present study, we show that mIL-6 expression also prompts microglial activation and increased expression of phagocytic receptors, lending further support for the idea that microglial phagocytosis plays a key role in the cytokine-stimulated clearance of $A \beta$ aggregates. 
A

Iba-1
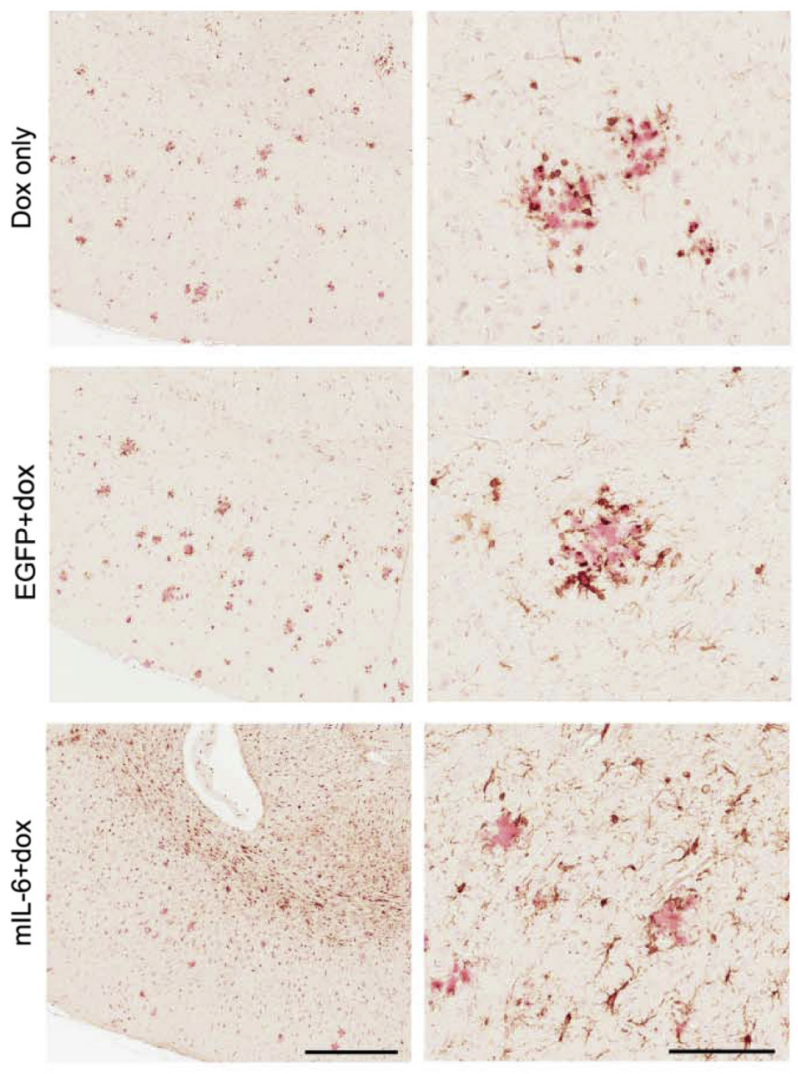

C

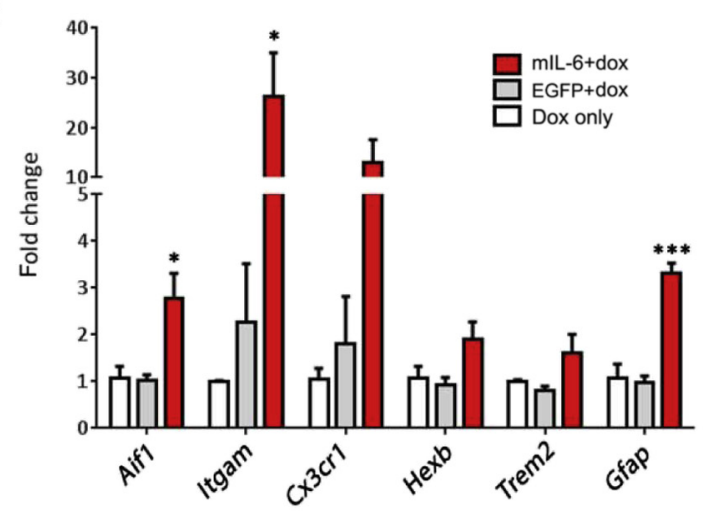

In our previous study, ${ }^{16}$ lifelong overexpression of IL-6 in APP mice was highly effective in preventing amyloid plaque deposition. When IL-6 expression was introduced into adult APP mice with existing disease, however, this intervention had only a small effect on subsequent plaque load. This difference suggests that once plaques are formed, mIL-6 activation becomes less effective for $\mathrm{A} \beta$ clearance because plaque-associated $\mathrm{A} \beta$ peptide may be less amenable to microglial uptake or by other clearance mechanisms. Alternatively, past a certain age the rate of deposition in this model may exceed the rate of clearance even after innate immune activation. By diminishing the rate of $A \beta$
B

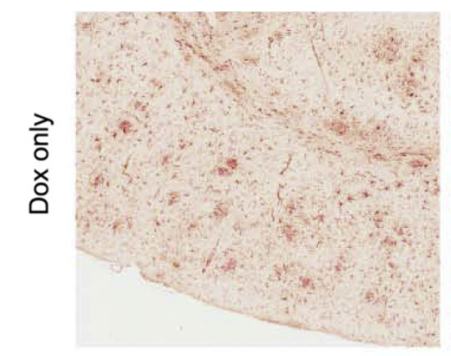

GFAP
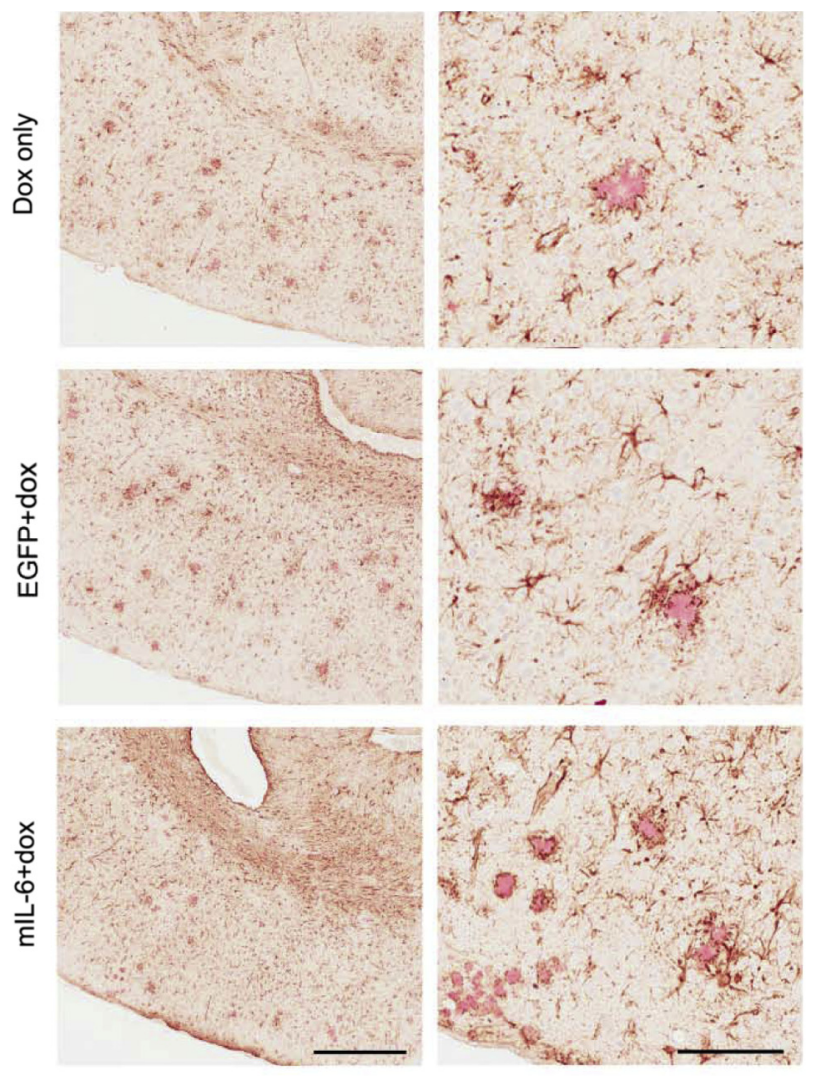

Figure 4 Adeno-associated virus 1 expressing murine IL-6 (AAV1mIL-6)-injected mice show enhanced reactive gliosis. A and B: Representative images of Congo red-stained amyloid plaques decorated with (A) anti-Iba-1 immunostained microglia or (B) anti-enhanced green fluorescent protein (GFAP)-immunostained astrocytes in each treatment group. Mice receiving mIL-6 + doxycycline (dox) show more widespread microglial and astrocytic activation in the parenchyma. C: Quantitative RT-PCR analysis of microglial markers showed increased mRNA expression of several microglial gene products and the astrocyte marker Gfap in the mIL- $6+$ dox group versus controls. Data are expressed as fold change from untreated control mice. ${ }^{*} P<0.05,{ }^{* *} P<0.001$ versus untreated controls. Scale bars: $500 \mu \mathrm{m}$ (A and B, left column); $100 \mu \mathrm{m}$ (A and B, right column).

production with the use of dox, we have rebalanced this equation by slowing plaque formation while simultaneously accelerating A $\beta$ clearance through local mIL-6 immune activation. Compared with dox or AAV1-mIL-6 expression alone, the combination of AAV1-mIL- 6 and A $\beta$ suppression was significantly more effective in attenuating amyloid deposition and removing diffuse amyloid plaques. Of note, however, even combination treatment was unable to remove fibrillar Thio-S-positive plaques, leaving the area of Thio-S staining unchanged from pretreatment levels. This finding suggests that once Thio-S plaques are formed, they are resistant to removal even by cytokine-activated microglial 
cells. Finally, in all treatment paradigms, total $A \beta$ plaque burden (diffuse + fibrillar) was higher at the end of the experiment than at the outset of treatment even in mice receiving combination treatment. We conclude that the main effect of our combination strategy was robust attenuation of ongoing amyloid deposition rather than removal of preexisting plaques.

Microglial cells are important regulators of central nervous system health. ${ }^{28-30}$ Recent genetic studies have identified heterozygous missense mutations in the microglial TREM2 receptor as risk factors for $\mathrm{AD}$ and other neurodegenerative disorders, highlighting a role for microglial dysfunction in pathogenesis. ${ }^{31,32}$ Microglial cells may also become senescent with aging, ${ }^{33}$ rendering them less able to remove toxic protein aggregates or apoptotic neurons from the brain. Modulating the microglial activation state may therefore be an attractive therapeutic approach for $\mathrm{AD}$ and other age-related neurodegenerative diseases. Before this treatment strategy is considered in the clinic, potential side effects associated with increased proinflammatory cytokine levels in the central nervous system, such as sickness behavior, altered synaptic function, and/or transient cognitive impairment, should be carefully evaluated. Although we do not see overt toxicity with mIL-6 expression in our studies, monitoring of these possible altered behavioral phenotypes and ways to alleviate these symptoms during treatment should be considered before the start of a clinical trial. Although direct AAV-mediated expression of cytokines in the brain may not be feasible in the clinic, the central neuroimmune axis might also be accessed by targeting peripheral immune cells. Several recent studies suggest that manipulating the peripheral immune system can alter central nervous system microglial responses in mouse models of AD. Systemic injections of the lipopolysaccharide-derived Toll-like receptor 4 agonist monophosphoryl lipid A elicited a phagocytic microglial response in the brain that attenuated plaque deposition and improved cognitive function in APP/PS1 mice. ${ }^{34}$ Similarly, peripheral stimulation with a Toll-like receptor 9 agonist, class B CpG (cytosine-phosphate-guanine) oligodeoxynucleotides, reduced both amyloid and tau pathologic disorder in $\operatorname{Tg} 2576$ and $3 \mathrm{x}-\mathrm{TgAD}$ mice ${ }^{35,36}$ and lowered cerebral amyloid angiopathy pathologic disorder in Tg-SwDI mice. ${ }^{37}$ Blocking the immune checkpoint programed death-1 pathway with peripheral injection of antiprogramed death-1 antibody promoted the recruitment of monocyte-derived macrophages to the brain, enhanced amyloid- $\beta$ plaque clearance, and improved cognitive outcomes in 5XFAD mice. ${ }^{38}$ Finally, transient depletion of Foxp3-positive regulatory $T$ cells $\left(T_{\text {regs }}\right)$ stimulated $A \beta$ plaque clearance and improved behavioral deficits in 5XFAD mice. ${ }^{39}$ Collectively, these studies strongly support the potential benefit of central neuroimmune activation in $\mathrm{AD}$.

Although we were unable to directly compare our genetic APP reduction with treatment combinations based on pharmacologic $\mathrm{A} \beta$ inhibitors such as BACE1 blockers, our findings nevertheless support further study using such combination strategies. Our results also reveal limitations to this approach. However, although our study does show significant attenuation of ongoing plaque formation, even combination treatment was unable to remove preexisting Thio-S-positive fibrillar plaques. Anti-A $\beta$ antibodies capable of directly binding Thio-S plaques may be needed to stimulate microglial removal of these fibrillar aggregates. ${ }^{40}$ Thus, an alternative approach might combine antiA $\beta$ immunotherapy with innate immune activation of microglial cells to maximize breakdown of deposited plaques. Additional study is clearly warranted. Our work advances the broader discussion of combination treatments by demonstrating that a dual-line approach allowed us to control plaque deposition in adult APP mice at an age when neither strategy alone was able. These results suggest that in the emerging clinical setting of secondary prevention for $\mathrm{AD}$, combination approaches should be given strong consideration for future trials because they may better curtail $\mathrm{A} \beta$ pathologic disorder and do so at lower doses for each line of treatment.

\section{Acknowledgments}

We thank Dennis Dickson, Monica Castanedes-Casey, Virginia Phillips, and Linda Rousseau for help with immunohistopathology; and Sarah Waters and Kristen Masudipoya for help with animal breeding and husbandry.

\section{References}

1. Hardy J, Selkoe DJ: The amyloid hypothesis of Alzheimer's disease: progress and problems on the road to therapeutics. Science 2002, 297: 353-356

2. Golde TE: The Abeta hypothesis: leading us to rationally-designed therapeutic strategies for the treatment or prevention of Alzheimer disease. Brain Pathol 2005, 15:84-87

3. Karran E, De Strooper B: The amyloid cascade hypothesis: are we poised for success or failure? J Neurochem 2016, 139 Suppl 2: 237-252

4. Jack CR Jr, Knopman DS, Jagust WJ, Shaw LM, Aisen PS, Weiner MW, Petersen RC, Trojanowski JQ: Hypothetical model of dynamic biomarkers of the Alzheimer's pathological cascade. Lancet Neurol 2010, 9:119-128

5. Jack CR Jr, Knopman DS, Jagust WJ, Petersen RC, Weiner MW, Aisen PS, Shaw LM, Vemuri P, Wiste HJ, Weigand SD, Lesnick TG, Pankratz VS, Donohue MC, Trojanowski JQ: Tracking pathophysiological processes in Alzheimer's disease: an updated hypothetical model of dynamic biomarkers. Lancet Neurol 2013, 12:207-216

6. Golde TE, Petrucelli L, Lewis J: Targeting Abeta and tau in Alzheimer's disease, an early interim report. Exp Neurol 2010, 223: 252-266

7. Golde TE, Schneider LS, Koo EH: Anti-abeta therapeutics in Alzheimer's disease: the need for a paradigm shift. Neuron 2011, 69: 203-213

8. Salloway S, Sperling R, Fox NC, Blennow K, Klunk W, Raskind M, Sabbagh M, Honig LS, Porsteinsson AP, Ferris S, Reichert M, Ketter N, Nejadnik B, Guenzler V, Miloslavsky M, Wang D, Lu Y, Lull J, Tudor IC, Liu E, Grundman M, Yuen E, Black R, Brashear HR; 
Bapineuzumab 301 and 302 Clinical Trial Investigators: Two phase 3 trials of bapineuzumab in mild-to-moderate Alzheimer's disease. $\mathrm{N}$ Engl J Med 2014, 370:322-333

9. Salloway S, Sperling R, Brashear HR: Phase 3 trials of solanezumab and bapineuzumab for Alzheimer's disease. N Engl J Med 2014, 370:1460

10. Arrighi HM, Barakos J, Barkhof F, Tampieri D, Jack C Jr, Melancon D, Morris K, Ketter N, Liu E, Brashear HR: Amyloid-related imaging abnormalities-haemosiderin (ARIA-H) in patients with Alzheimer's disease treated with bapineuzumab: a historical, prospective secondary analysis. J Neurol Neurosurg Psychiatry 2016, 87:106-112

11. De Strooper B, Chavez Gutierrez L: Learning by failing: ideas and concepts to tackle gamma-secretases in Alzheimer's disease and beyond. Annu Rev Pharmacol Toxicol 2015, 55:419-437

12. Sperling RA, Rentz DM, Johnson KA, Karlawish J, Donohue M, Salmon DP, Aisen P: The A4 study: stopping AD before symptoms begin? Sci Transl Med 2014, 6:228fs13

13. Sevigny J, Chiao P, Bussiere T, Weinreb PH, Williams L, Maier M, Dunstan R, Salloway S, Chen T, Ling Y, O'Gorman J, Qian F, Arastu M, Li M, Chollate S, Brennan MS, Quintero-Monzon O, Scannevin RH, Arnold HM, Engber T, Rhodes K, Ferrero J, Hang Y, Mikulskis A, Grimm J, Hock C, Nitsch RM, Sandrock A: The antibody aducanumab reduces Abeta plaques in Alzheimer's disease. Nature 2016, 537:50-56

14. Mullard A: BACE inhibitor bust in Alzheimer trial. Nat Rev Drug Discov 2017, 16:155

15. Sala Frigerio C, De Strooper B: Alzheimer's disease mechanisms and emerging roads to novel therapeutics. Annu Rev Neurosci 2016, 39: $57-79$

16. Chakrabarty P, Jansen-West K, Beccard A, Ceballos-Diaz C, Levites Y, Verbeeck C, Zubair AC, Dickson D, Golde TE, Das P: Massive gliosis induced by interleukin-6 suppresses Abeta deposition in vivo: evidence against inflammation as a driving force for amyloid deposition. FASEB J 2010, 24:548-559

17. Chakrabarty P, Ceballos-Diaz C, Beccard A, Janus C, Dickson D, Golde TE, Das P: IFN-gamma promotes complement expression and attenuates amyloid plaque deposition in amyloid beta precursor protein transgenic mice. J Immunol 2010, 184:5333-5343

18. Chakrabarty P, Herring A, Ceballos-Diaz C, Das P, Golde TE: Hippocampal expression of murine TNFalpha results in attenuation of amyloid deposition in vivo. Mol Neurodegener 2011, 6:16

19. Jankowsky JL, Slunt HH, Gonzales V, Savonenko AV, Wen JC, Jenkins NA, Copeland NG, Younkin LH, Lester HA, Younkin SG, Borchelt DR: Persistent amyloidosis following suppression of Abeta production in a transgenic model of Alzheimer disease. PLoS Med 2005, 2:e355

20. Mayford M, Bach ME, Huang YY, Wang L, Hawkins RD, Kandel ER: Control of memory formation through regulated expression of a CaMKII transgene. Science 1996, 274:1678-1683

21. Chakrabarty P, Li A, Ceballos-Diaz C, Eddy JA, Funk CC, Moore B, DiNunno N, Rosario AM, Cruz PE, Verbeeck C, Sacino A, Nix S, Janus C, Price ND, Das P, Golde TE: IL-10 alters immunoproteostasis in APP mice, increasing plaque burden and worsening cognitive behavior. Neuron 2015, 85:519-533

22. Moore BD, Chakrabarty P, Levites Y, Kukar TL, Baine AM, Moroni T, Ladd TB, Das P, Dickson DW, Golde TE: Overlapping profiles of Abeta peptides in the Alzheimer's disease and pathological aging brains. Alzheimers Res Ther 2012, 4:18

23. Ehlers MR: CR3: a general purpose adhesion-recognition receptor essential for innate immunity. Microbes Infect 2000, 2:289-294

24. Zhang Y, Chen K, Sloan SA, Bennett ML, Scholze AR, O'Keeffe S, Phatnani HP, Guarnieri P, Caneda C, Ruderisch N, Deng S, Liddelow SA, Zhang C, Daneman R, Maniatis T, Barres BA, Wu JQ: An RNA-sequencing transcriptome and splicing database of glia, neurons, and vascular cells of the cerebral cortex. J Neurosci 2014, 34 11929-11947

25. Butovsky O, Jedrychowski MP, Moore CS, Cialic R, Lanser AJ, Gabriely G, Koeglsperger T, Dake B, Wu PM, Doykan CE, Fanek Z,
Liu L, Chen Z, Rothstein JD, Ransohoff RM, Gygi SP, Antel JP, Weiner HL: Identification of a unique TGF-beta-dependent molecular and functional signature in microglia. Nat Neurosci 2014, 17:131-143

26. Wang A, Das P, Switzer RC III, Golde TE, Jankowsky JL: Robust amyloid clearance in a mouse model of Alzheimer's disease provides novel insights into the mechanism of amyloid-beta immunotherapy. J Neurosci 2011, 31:4124-4136

27. Shaftel SS, Kyrkanides S, Olschowka JA, Miller JN, Johnson RE, O'Banion MK: Sustained hippocampal IL-1 beta overexpression mediates chronic neuroinflammation and ameliorates Alzheimer plaque pathology. J Clin Invest 2007, 117:1595-1604

28. Gomez-Nicola D, Perry VH: Microglial dynamics and role in the healthy and diseased brain: a paradigm of functional plasticity. Neuroscientist 2015, 21:169-184

29. Parkhurst CN, Yang G, Ninan I, Savas JN, Yates JR III, Lafaille JJ, Hempstead BL, Littman DR, Gan WB: Microglia promote learningdependent synapse formation through brain-derived neurotrophic factor. Cell 2013, 155:1596-1609

30. Crotti A, Ransohoff RM: Microglial Physiology and Pathophysiology: insights from Genome-wide Transcriptional Profiling. Immunity 2016 , 44:505-515

31. Guerreiro R, Wojtas A, Bras J, Carrasquillo M, Rogaeva E, Majounie E, Cruchaga C, Sassi C, Kauwe JS, Younkin S, Hazrati L, Collinge J, Pocock J, Lashley T, Williams J, Lambert JC, Amouyel P, Goate A, Rademakers R, Morgan K, Powell J, St George-Hyslop P, Singleton A, Hardy J: TREM2 variants in Alzheimer's disease. N Engl J Med 2013, 368:117-127

32. Jonsson T, Stefansson H, Steinberg S, Jonsdottir I, Jonsson PV, Snaedal J, Bjornsson S, Huttenlocher J, Levey AI, Lah JJ, Rujescu D, Hampel H, Giegling I, Andreassen OA, Engedal K, Ulstein I, Djurovic S, IbrahimVerbaas C, Hofman A, Ikram MA, van Duijn CM, Thorsteinsdottir U, Kong A, Stefansson K: Variant of TREM2 associated with the risk of Alzheimer's disease. N Engl J Med 2013, 368:107-116

33. Streit WJ, Xue QS: Human CNS immune senescence and neurodegeneration. Curr Opin Immunol 2014, 29:93-96

34. Michaud JP, Halle M, Lampron A, Theriault P, Prefontaine P, Filali M, Tribout-Jover P, Lanteigne AM, Jodoin R, Cluff C, Brichard V, Palmantier R, Pilorget A, Larocque D, Rivest S: Toll-like receptor 4 stimulation with the detoxified ligand monophosphoryl lipid A improves Alzheimer's disease-related pathology. Proc Natl Acad Sci U S A 2013, 110:1941-1946

35. Scholtzova H, Kascsak RJ, Bates KA, Boutajangout A, Kerr DJ, Meeker HC, Mehta PD, Spinner DS, Wisniewski T: Induction of tolllike receptor 9 signaling as a method for ameliorating Alzheimer's disease-related pathology. J Neurosci 2009, 29:1846-1854

36. Goni F, Herline K, Peyser D, Wong K, Ji Y, Sun Y, Mehta P, Wisniewski T: Immunomodulation targeting of both Abeta and tau pathological conformers ameliorates Alzheimer's disease pathology in TgSwDI and 3xTg mouse models. J Neuroinflammation 2013, 10:150

37. Scholtzova H, Do E, Dhakal S, Sun Y, Liu S, Mehta PD, Wisniewski T: Innate immunity stimulation via Toll-like receptor 9 ameliorates vascular amyloid pathology in Tg-SwDI mice with associated cognitive benefits. J Neurosci 2017, 37:936-959

38. Baruch K, Deczkowska A, Rosenzweig N, Tsitsou-Kampeli A, Sharif AM, Matcovitch-Natan O, Kertser A, David E, Amit I, Schwartz M: PD-1 immune checkpoint blockade reduces pathology and improves memory in mouse models of Alzheimer's disease. Nat Med 2016, 22:135-137

39. Baruch K, Rosenzweig N, Kertser A, Deczkowska A, Sharif AM, Spinrad A, Tsitsou-Kampeli A, Sarel A, Cahalon L, Schwartz M: Breaking immune tolerance by targeting Foxp3(+) regulatory T cells mitigates Alzheimer's disease pathology. Nat Commun 2015, 6:7967

40. Demattos RB, Lu J, Tang Y, Racke MM, Delong CA, Tzaferis JA, Hole JT, Forster BM, McDonnell PC, Liu F, Kinley RD, Jordan WH, Hutton ML: A plaque-specific antibody clears existing beta-amyloid plaques in Alzheimer's disease mice. Neuron 2012, 76:908-920 